\title{
INTERAÇÃO ENXERTO E PORTA-ENXERTO NA INCIDÊNCIA DA RESINOSE DO CAJUEIRO ${ }^{1}$
}

\author{
JOSÉ EMILSON CARDOSO, JOSÉ JAIME VASCONCELOS CAVALCANTI, \\ ALEX QUEIROZ CYSNE, TOMIL RICARDO MAIA DE SOUSA, \\ MÁRCIO CLEBER DE MEDEIROS CORRÊA
}

RESUMO - A expansão do cajueiro-anão-precoce no Brasil vem promovendo baixa diversidade genética dos genótipos usados como enxerto e porta-enxerto, ensejando vulnerabilidade da cultura às vicissitudes bióticas como a resinose (Lasiodiplodia theobromae), principal doença do cajueiro no semiárido nordestino. Estima-se que cerca de meio milhão de mudas de cajueiro-anão-precoce sejam produzidas anualmente, sendo a grande maioria delas produzida de propágulos enxertados sobre plântulas oriundas de sementes do clone CCP 06. A principal estratégia de manejo da resinose é o plantio de clones resistentes. Entretanto, entre os aspectos que contribuem para sucessivas epidemias da resinose, destaca-se a falta de estudos de interações genotípicas entre enxerto e porta-enxerto. Este estudo objetivou avaliar o efeito de combinações de clones de cajueiro-anão-precoce usados como enxerto e porta-enxerto na incidência da resinose. Sementes de plantas de polinização aberta de seis clones selecionados foram usadas para produção de porta-enxerto. Os clones comerciais CCP 76 (suscetível) e BRS 226 (resistente) foram usados como enxertos. O experimento foi realizado obedecendo ao delineamento de blocos ao acaso, no esquema fatorial 2 × 6 , com quatro repetições e nove plantas por parcela do tipo quadrada, sob condições de elevada pressão da doença, em Pio IX-PI. Três anos após o plantio definitivo, a incidência $(I)$ e a severidade $(S)$ (escala de 0 a 4 ) da resinose foram superiores no clone CCP 76 ( $I=97 \%$ e $S=2,10)$ em relação ao BRS 226 ( $I=33 \%$ e $S=0,36)$, independentemente do porta-enxerto. O clone CP 06 como porta-enxerto foi o único que reduziu a incidência e a severidade da resinose, independentemente do genótipo do enxerto. Este clone foi capaz de reduzir a severidade da resinose do clone CCP $76(S=2,10)$ como enxerto em quase $30 \%$.

Termos para indexação: Lasiodiplodia theobromae, Anacardium occidentale, resistência genética.

\section{EFFECT OF CASHEW CLONE USED AS ROOTSTOCKS AND SCIONS ON GUMMOSIS INCIDENCE IN CASHEW PLANTS}

\begin{abstract}
The expansion of early dwarf cashew (EDC) crop in Brazil has promoted a reduced genetic diversity of cashew genotypes used to both as scion and root-stock. This may contribute to enhance crop vulnerability to some biological harm such as the infection by the gummosis fungus agent, Lasiodiplodia theobromae, the most important disease of this plant in semi-arid north-eastern Brazil. It has been estimated that half a million graft plants of EDC are yearly produced in Brazil, most of them obtained by grafting over seedling from CCP 06 clone as root-stock. The only means of managing gummosis is by the use of genetically resistant clones. However, the lack of studies on the scion and stock genotypic interactions has contributed to outbreak of successive gummosis epidemics. This study was developed in order to evaluate the effect of different combinations of EDC clones used as scion and stock on gummosis incidence. Rootstocks, prepared from seeds of six open pollinated plants, were grafted with two commercial clones: CCP 75 (susceptible) and BRS 226 (resistant). The field trial was laid out under high disease pressure, in a 2 by 6 factorial scheme in a completely randomized block design with four replications and nine plants per plot. Disease incidence $(I)$ and severity $(S)$ (scale 0 to 4 ) was monitored for three years. On the third year, gummosis incidence and severity were higher in CCP $76(I=97 \%$ and $I=2.10)$ than in BRS $226(I=33 \%$ and $I=0.36$ ), independently of root-stock. CCP 06 clone was the only one root-stock which reduced both incidence and severity of gummosis independently of scion. This clone was able to reduce susceptibility of CCP 76 as canopy.
\end{abstract}

Index terms: Lasiodiplodia theobromae, Anacardium occidentale, genetic resistance.

\footnotetext{
(Trabalho 176-09). Recebido em: 28-07-2009. Aceito para publicação em: 13-05-2010. Trabalho desenvolvido com recursos do Edital CNPq/MAPA/SDA No 064/2008

${ }^{2}$ Eng. Agr., PhD., Embrapa Agroindústria Tropical, Caixa Postal 3761, CEP 60511-110 Fortaleza-CE. E-mail: emilson@cnpat.embrapa.br; jaime@cnpat.embrapa.br; bordallo@cnpat.embrapa.br

${ }^{3}$ Eng. Agr., Embrapa Amazônia Ocidental, Manaus-AM. E-mail:

${ }^{4}$ Eng. Agr., Mestrando em Fitotecnia da Universidade Federal do Ceará, Fortaleza-CE. E-mail: tomilagroufc@gmail.com

${ }^{5}$ Eng. Agr., DSc. Professor da Universidade Federal do Ceará, CEP 60451-970 Fortaleza-CE. E-mail: mcleber@ufc.br
} 


\section{INTRODUÇÃO}

A cultura do cajueiro vem experimentando acentuado crescimento, tanto em área de cultivo como, principalmente, a nível tecnológico, face à modernização das técnicas de manejo. A propagação agâmica viabilizou o plantio de clones selecionados do tipo cajueiro-anão-precoce. Com isso, estima-se que cerca de meio milhão de mudas de cajueiro-anãoprecoce sejam produzidas anualmente no Nordeste (CAVALCANTE JUNIOR et al., 2002). Entretanto, quase todas essas mudas são produzidas usando somente um único genótipo como porta-enxerto (cajueiro CCP 06), sobre o qual informações publicadas de estudos da interação com patógenos e com clone do enxerto são inexistentes. Além do caráter técnico, essa condição evidencia também um problema de natureza logística decorrente da baixa oferta de cultivares de cajueiro-anão-precoce no fornecimento de sementes para produção de porta-enxerto (CAVALCANTE JUNIOR et al., 2002).

A importância do porta-enxerto reveste-se de maior influência ao se relacionar com a reação do cajueiro a doenças transmitidas pela muda como a resinose, doença causada pelo fungo Lasiodiplodia theobromae (Pat.) Griff \& Maubl., hoje a principal doença do cajueiro no Semiárido Nordestino. Essa doença é comumente observada em plantas submetidas a estresses. As plantas acometidas pela resinose têm a transpiração alterada decorrente da redução da condutância estomática e da fotossíntese líquida (BEZERRA et al., 2003). L. theobromae é capaz de colonizar tecidos de plantas sem exibir sintomas de infecção (MOHALI et al., 2005, MULLEN et al., 1991; RUBINI et al., 2005), possibilitando sua disseminação através de sementes, propágulos vegetativos e porta-enxertos, de forma despercebida (CARDOSO et al., 1998; CARDOSO et al., 2004). Acredita-se que essa característica, certamente, é a maior responsável pelas epidemias observadas na microrregião do sudeste do Piauí (CARDOSO et al., 1998; CARDOSO et al., 2004; FREIRE; CARDOSO, 2002).

Os genótipos de cajueiro apresentam diferenças na reação quanto à resinose, ensejando a seleção de indivíduos resistentes como BRS 226-Planalto e Embrapa 51, ambos selecionados na região semiárida (PAIVA, et al., 2002; CARDOSO et al., 2006 e 2007). Entretanto, entre os aspectos que contribuem para a ocorrência de severas epidemias da resinose no Semiárido Nordestino, destacam-se a expansão do cultivo com uso do clone suscetível "CCP 76", a falta de estudos epidemiológicos relacionados ao manejo integrado, incluindo-se estudos de interações genotípicas do enxerto e porta-enxerto e a falta de um método de detecção precoce da presença do fungo nos propágulos usados para a produção da muda.

A enxertia é uma técnica que tem sido usada largamente no controle de doenças causadas por patógenos do solo e doenças que afetam o sistema vascular, como a morte súbita dos citros (BASSANEZI et al., 2002; GIRARDI et al., 2007; SHAKED et al., 1987; MORENO et al., 1994; POMPEU JUNIOR, 2005), fusariose e morte prematura do maracujazeiro (CHAVES et al., 2004; PACE, 1984; RUGGIERO, 2000; SÃO JOSÉ, 1991; MELETTI; BRUCKNER, 2001; MENEZES et al. 1994), as murchas de Fusarium e Monosporascus e o cancro gomoso em cucurbitáceas (BLETSOS, 2005; COHEN et al., 2002), podridão radicular de Fusarium em feijão-comum (CICHY et al. 2007). A estratégia mais comum consiste no uso do porta-enxerto resistente à doença, enxertado com o genótipo comercial.

O objetivo deste estudo foi avaliar o efeito de combinações de clones de cajueiro-anão-precoce usados como enxerto e porta-enxerto na incidência da resinose.

\section{MATERIAL E MÉTODOS}

O estudo foi conduzido em um pomar comercial da Fazenda Planalto (latitude $6^{\circ} 43^{\prime} \mathrm{S}$, longitude $40^{\circ} 35^{\prime} \mathrm{W} \mathrm{Gr}$, altitude $730 \mathrm{~m}$ ), localizada no município de Pio IX-PI. O solo da área do pomar é um Latossolo Amarelo álico, com pH 4,5 na camada de $0-20 \mathrm{~cm}$. As mudas foram produzidas no viveiro da própria fazenda, utilizando-se de sementes de plantas de polinização aberta de seis genótipos selecionados (Figura 2). O clone CCP 06 foi usado como referência de recomendação geral no Brasil, enquanto os outros cinco (CCP 09, CCP 76, CAC 38, CAC 40 e BRS 226) foram selecionados com base na adaptabilidade na região e na tolerância à salinidade (dados não publicados). A enxertia foi feita 90 dias após o plantio, empregando-se o método de garfagem em fenda cheia. Os garfos dos dois clones (CCP 76 e BRS 226) foram selecionados de jardins clonais da própria fazenda. As mudas foram produzidas em sacos de polietileno preto $(15 \mathrm{~cm}$ de largura por $28 \mathrm{~cm}$ de altura) e transplantadas 120 dias após a semeadura. O plantio foi realizado em sistema retangular, no espaçamento de $8 \mathrm{~m} \times 6 \mathrm{~m}$, numa área de 3,7 ha. As covas, com dimensões de 40 x 40 x 40 $\mathrm{cm}$, foram adubadas, conforme recomendação baseada na análise do solo $(\mathrm{pH}=4,3 ; \mathrm{Ca}=2,1 \mathrm{mmolc} /$ $\mathrm{dm}^{3} ; \mathrm{Mg}=0,5 \mathrm{mmolc} / \mathrm{dm}^{3} ; \mathrm{K}=0,2 \mathrm{mmolc} / \mathrm{dm}^{3} ; \mathrm{CTC}$ $=19,1 \mathrm{mmolc} / \mathrm{dm}^{3}$; saturação de bases $13,7 \%$ ) em pré-plantio com $100 \mathrm{~g}$ de calcário dolomítico (PRNT $80 \%), 400 \mathrm{~g}$ de superfosfato simples $\left(72 \mathrm{~g}\right.$ de $\left.\mathrm{P}_{2} \mathrm{O}_{5}\right)$ 
e $40 \mathrm{~g}$ de FTE BR (AQUINO et al., 2002).

O delineamento usado foi em blocos ao acaso, no esquema fatorial $2 \times 6$ (enxerto x portaenxerto), com 4 repetições e 9 plantas por parcela do tipo quadrada. Uma bordadura simples foi usada em todo o contorno do experimento. $\mathrm{O}$ experimento foi instalado no campo, em março de 2005.

Os tratos culturais consistiram no tutoramento das plantas no campo, gradagem mecânica anual (entre os meses de agosto e setembro) e pulverizações ocasionais com os inseticidas Fenitrothion e Dimetoato para o controle da broca-das-pontas (Anthistarcha binocularis) e da mosca-branca (Aleurodicus cocois), respectivamente. Nenhuma poda foi realizada durante o período de avaliação.

As plantas foram numeradas e avaliadas quanto à resinose, 3 anos após o plantio definitivo. A severidade da resinose $(S)$ foi estimada de acordo com a escala descritiva desenvolvida por Cardoso et al. (2002), que consiste em notas de 0 a 4 , sendo 0 - sem sintomas; 1 - pequenos e poucos cancros, rachaduras pequenas sem exsudação de goma; 2- cancros maiores, espalhados pelos ramos ou no tronco, rachaduras acentuadas ( $1 / 3$ da circunferência) com exsudação; 3 - cancros maiores que $1 / 3$ da circunferência com abundante exsudação; 4- cancro atingindo toda a circunferência do tronco, descoloração, amarelecimento e/ou seca do(s) ramo(s) acima da área afetada com intensa exsudação. A produção de castanhas por parcela também foi avaliada no terceiro e quarto anos após o plantio (2007 e 2008).

Os dados de incidência $(I=$ proporção de plantas doentes por parcela), severidade $\left[\left(S=\sum\left(x_{\mathrm{i}} \cdot \mathrm{n}_{\mathrm{i}}\right) / n ;\right.\right.$ em que: $x$ grau de doença na escala de 0 a $4, n_{i}$ representa a frequência de plantas por cada nota na escala e $n$ é o total de plantas doentes] e produção foram submetidos à análise de variância após transformação em $\sqrt{X+0,5}$, uma vez que a pressuposição de normalidade do desvio $\left(\varepsilon_{i j k}\right)$ não foi satisfeita pelo teste de Shapiro-Wilk A análise de variância obedeceu ao modelo estatístico:

$$
Y_{j i k}=\mu+B_{j}+E_{i}+P E_{k}+(E-P E)_{j k}+\varepsilon_{i j k}, \mathrm{em}
$$

que $Y_{j i k}$ é o valor observado nas ordens $j$ (blocos), $i$ (enxertos) e $k$ (porta-enxertos); $\mu$ é a média da população e $B_{j}, E_{i}, P E_{k},(E-P E)_{j k}$ e $\varepsilon_{i j k}$ são os efeitos de bloco, enxerto, porta-enxerto, interação enxerto/ porta-enxerto e erro, respectivamente. As médias foram comparadas pelo teste de Tukey $(\mathrm{P}=0,05)$. As análises estatísticas foram feitas usando o pacote estatístico SAS.

\section{RESULTADOS E DISCUSSÃO}

Os primeiros sintomas de resinose foram observados em quatro plantas isoladamente, em agosto de 2007, quando as mesmas atingiram a primeira safra, confirmando resultados anteriores (CARDOSO et al., 2004). Somente as plantas enxertadas com o clone CCP 76 foram afetadas nesse ano pela doença.

Em 2008, três anos após o plantio definitivo, a incidência e a severidade da resinose foram significativas $(\mathrm{P}<0,05)$, sendo superiores para o clone CCP $76(I=97 \%$ e $S=2,10)$ em relação ao BRS 226 ( $I=33 \%$ e $S=0,36$ ), independentemente do portaenxerto (Figura 1).

$\mathrm{Na}$ análise de variância dos dados de severidade da resinose, observou-se efeito significativo do enxerto e do porta-enxerto $(\mathrm{P}<0,01)$. Entretanto, a interação enxerto e porta-enxerto não foi significativa $(\mathrm{P}=0,13)$. Esse efeito não significativo da interação entre os fatores pode ter sido em decorrência do fato de que apenas um porta-enxerto $(\mathrm{CCP}$ 06) entre os seis testados tenha contribuído para a alteração no desempenho do enxerto na reação à resinose (Figura 2).

O clone CCP 06, como porta-enxerto, foi o único que teve efeito significativo $(\mathrm{P}=0,05)$ na redução da incidência e da severidade da resinose (Figura $3)$. Esse clone foi capaz de reduzir a severidade da resinose do clone CCP $76(S=2,10)$, como enxerto, em quase $30 \%$, pois a severidade da resinose no clone CCP 76, quando enxertado nesse clone, foi de 1,54 . As interpretações desses resultados sugerem o potencial desse clone no manejo da resinose e o forte indicativo da sua resistência a essa doença. Apesar do uso tradicional de sementes do clone CCP 06 para obtenção de porta-enxerto de cajueiro-anãoprecoce, mesmo nas regiões de elevada incidência da resinose, nenhuma referência da sua reação à mesma foi publicada. As características comerciais do CCP 06, principalmente quanto ao tamanho do fruto, são tidas como responsáveis pela sua não adoção como cultivar-copa nos plantios comerciais brasileiros, sendo cultivado somente como banco de sementes para a produção de porta-enxerto. A comprovação da resistência desse clone à resinose como copa, constitui-se em importante informação a ser buscada, a fim de adotá-lo como opção de fonte de resistência para futuros programas de melhoramento genético, visando à obtenção de genótipos superiores para plantio comercial nas regiões de potenciais epidemias.

Estudos têm demonstrado que o agente causal da resinose pode ser disseminado endofiticamente ou a partir de plantas doentes, via agentes 
bióticos e abióticos, (CARDOSO et al., 1998; FREIRE; CARDOSO, 2002), sendo as mudas fonte de inóculo primário, e as plantas infectadas, como fonte secundária. Alguns autores referem-se a esse fenômeno como endofitismo (FAETH, 2002; AZEVEDO, 1998) enquanto que outros simplesmente o atribuem apenas ao estágio de patógeno latente (BAIRD; CARLING, 1998; CILLIERS et al., 1993, MÜLLEN et al. 1991). Baseado nessas informações, a possibilidade de que, por alguma razão ainda desconhecida, $L$. theobromae tenha baixa capacidade de sobreviver de forma latente em plantas do clone CCP 06, constitui-se numa hipótese a ser avaliada. Confirmada essa hipótese, a redução da doença obtida com esse clone deverá diminuir com o progresso da epidemia nos anos seguintes, uma vez que esse clone, como porta-enxerto, contribuiria apenas na redução do inóculo inicial.
Os dados obtidos das reações de resistência do clone BRS 226 e da suscetibilidade do CCP 76 (Figura 1) estão em consonância com resultados publicados anteriormente (CARDOSO et al., 2005; PAIVA et al., 2008), independentemente de qualquer um dos seis porta-enxertos usados. A produção do clone BRS 226 foi $46 \%$ significativamente superior ao do clone CCP 76, independentemente do porta-enxerto. Essa maior produção pode ser atribuída à resistência_à resinose obtida com esse clone. Analisando-se os dados de produção por porta-enxerto, surpreende-se o fato de que, mesmo tendo contribuído para a redução da resinose, com o clone CCP 06, ocorreu também uma redução significativa $(\mathrm{P}<0,05)$ em relação aos clones $\mathrm{CCP}$ 09 e BRS 226 (Figura 3). Uma provável explicação para essa queda de produção decorre de mudanças nas rotas metabólicas menos eficientes relacionadas à resistência. Estudos posteriores sobre os mecanismos de resistência desse genótipo são necessários para a elucidação desse fenômeno.
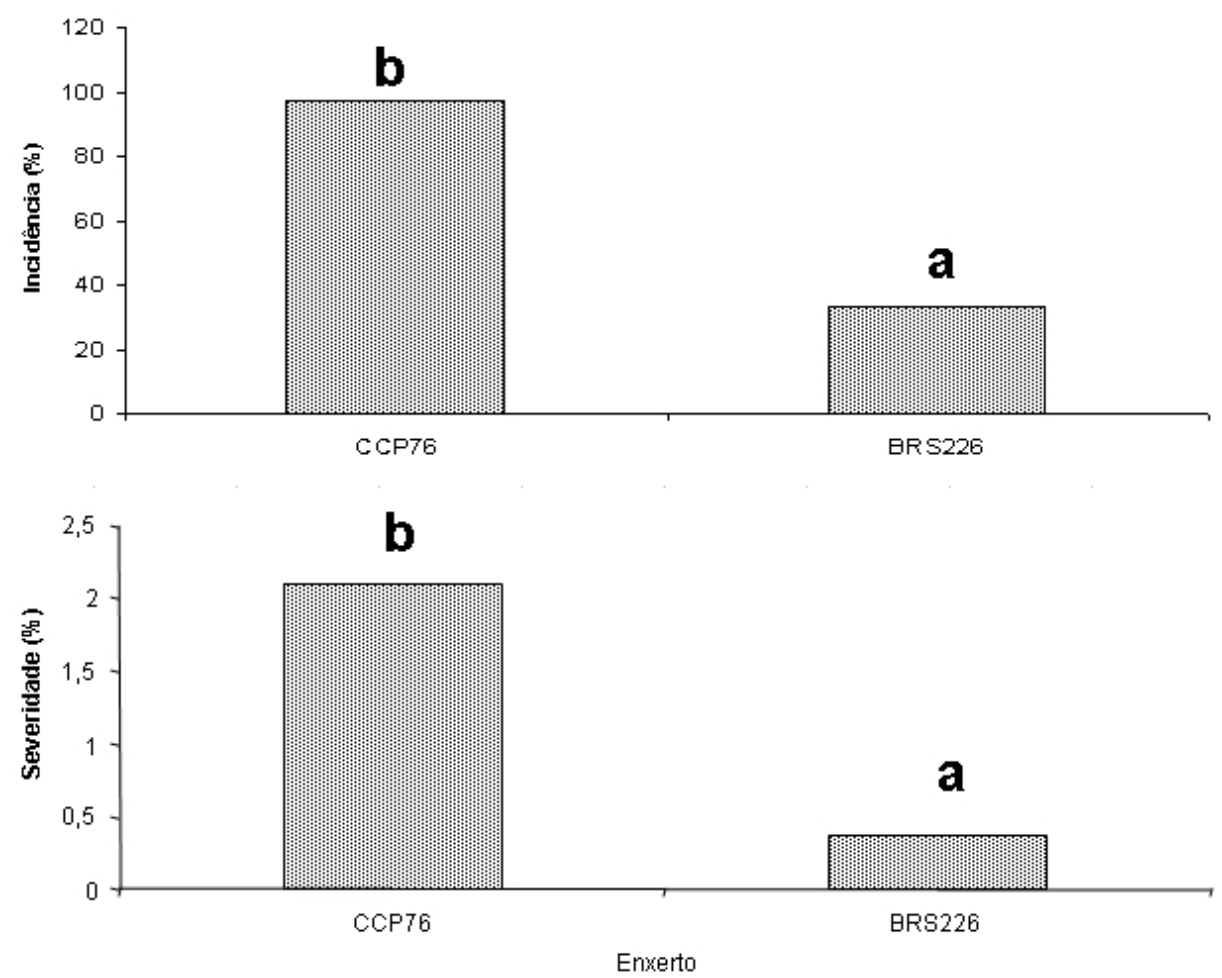

FIGURA 1 - Incidência (\%) e severidade $(S)$ da resinose do cajueiro (Lasiodiplodia theobromae (Pat.) Griff \& Maubl.), conforme uma escala de notas variando de 0 a 4, nos clones CCP 76 e BRS 226. Barras representam medias de 24 parcelas com nove plantas cada, enxertadas em seis diferentes porta-enxertos. Pio IX, Piauí, 2008. Barras contendo letras iguais não diferem estatisticamente, segundo o teste de Tukey $(\mathrm{p}=0,05)$. 


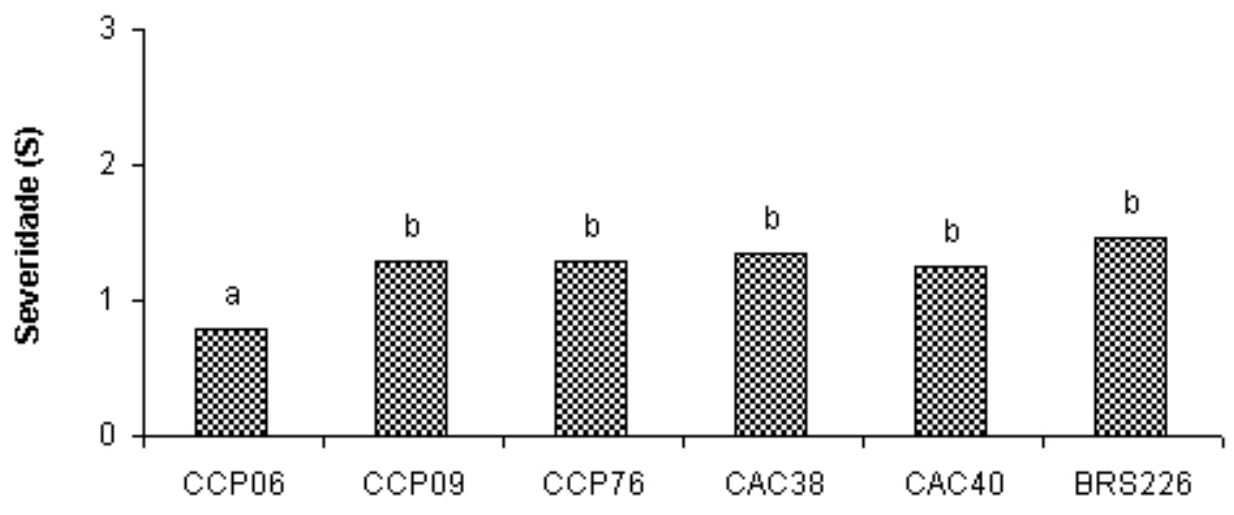

Porta-enxerto

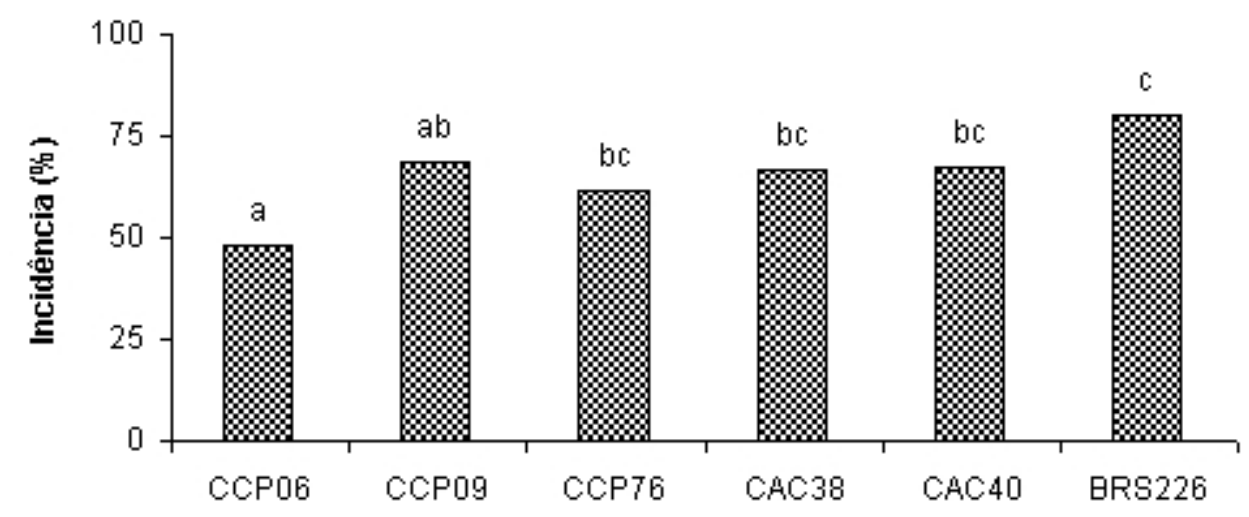

FIGURA 2 - Efeito do clone usado como porta-enxerto na incidência e severidade da resinose do cajueiro (Lasiodiplodia theobromae (Pat.) Griff \& Maubl.). A estimativa da severidade obedeceu a uma escala de notas variando de 0 a 4. Pio IX, Piauí, 2008. Barras representam médias de 4 repetições com nove plantas cada, enxertadas com dois diferentes clones (CCP 76 e BRS 226). Barras contendo letras iguais não diferem estatisticamente, segundo o teste de Tukey $(p=0,05)$. 


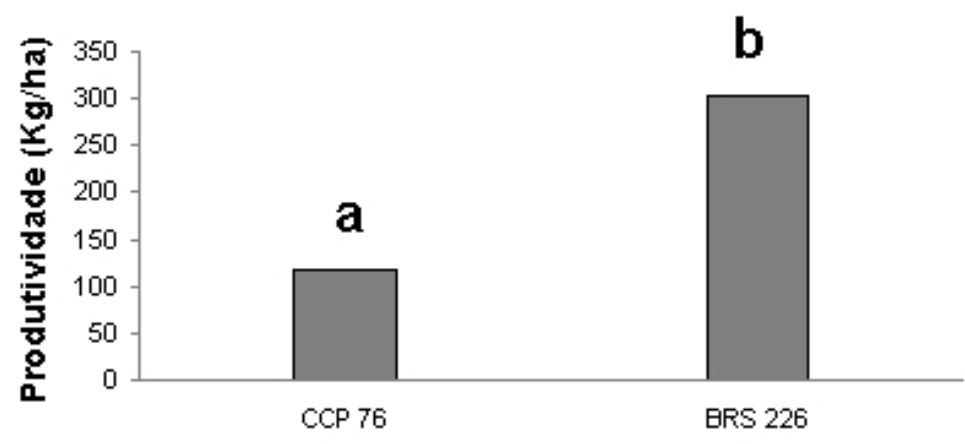

Enxerto

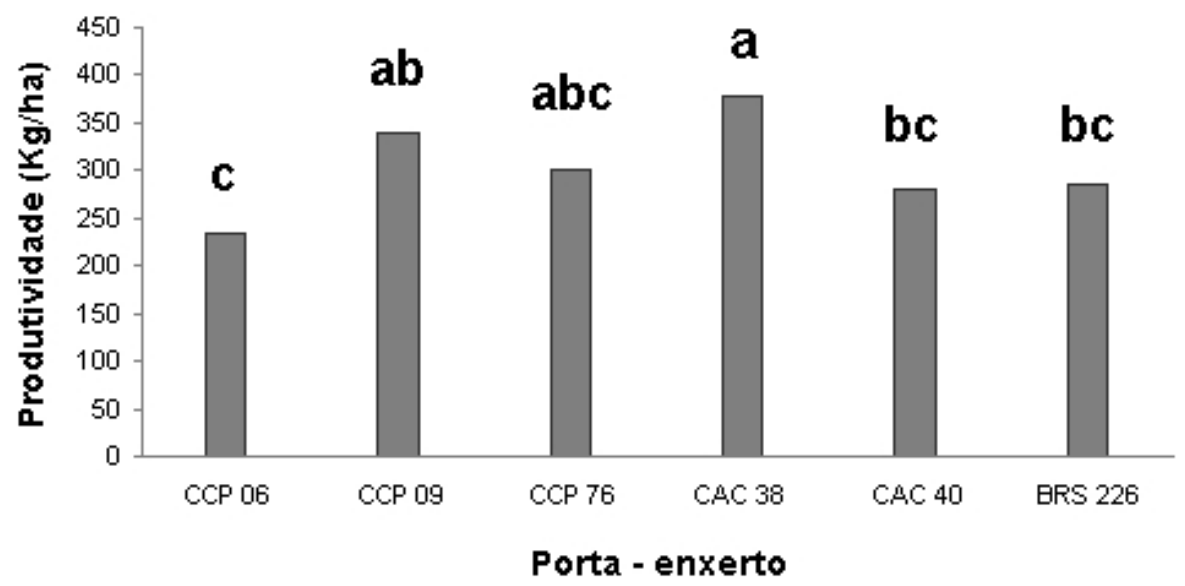

FIGURA 3 - Efeito do clone usado como enxerto (CCP 76 e BRS 226) e como porta-enxerto na produção de castanhas de caju (kg/ha). Pio IX, Piauí, 2008. Barras representam médias de 4 repetições com nove plantas cada. Barras contendo letras iguais não diferem estatisticamente, segundo o teste de Tukey $(\mathrm{p}=0,05)$.

\section{CONCLUSÕES}

1- O clone CCP 06, como porta-enxerto, reduz a incidência e a severidade da resinose do cajueiro, independentemente da reação do enxerto.

2- O clone BRS 226 é resistente à resinose, independentemente do porta-enxerto.

3- Não existe interação entre porta-enxerto e enxerto na incidência da resinose.

\section{AGRADECIMENTOS}

Os autores agradecem à direção da Companhia Industrial de Óleos do Nordeste (CIONE) e ao pessoal da Fazenda Planalto pela cessão da área experimental e apoio logístico durante a realização deste trabalho.

\section{REFERÊNCIAS}

AQUINO, A. R. L.; LIMA, A. A. C.; OLIVEIRA, F. N. S. Correção do solo e adubação. In: Barros, L. M. (Ed.). Caju produção: aspectos técnicos. Fortaleza: Embrapa Agroindústria Tropical, 2002. p. 38-42. (Frutas do Brasil, 30). 
AZEVEDO, J. L. Microorganismos endofíticos. In: MELO, I.S.; AZEVEDO, J. L. (Ed.). Ecologia microbiana. Jaguariúna: Editora EMBRAPA, 1998. p. 117-137.

BAIRD, R.; CARLING, D. Survival of parasitic anal saprophitic fingi or intact senescent colton roots. The Journal of Cotton Science, Cordova, v.2, p. 27-34, 1998.

BASSANEZI, R. B.; YAMAMOTO, P. T.; GIMENES-FERNANDES, N. Progresso dos sintomas de "morte súbita" em pomares de laranjeiras 'Valência' e 'Pêra'. In: CONGRESSO PAULISTA DE FITOPATOLOGIA, 25., 2002, Espírito Santo do Pinhal. Programa e resumos... p. 80 .

BEZERRA, M. A., CARDOSO, J. E., SANTOS, A. A., VIDAL, J. C.; ALENCAR, E. S. Efeito da resinose na fotossíntese do cajueiro anão precoce. Fortaleza: Embrapa/CNPAT, 2003. 12p. (Boletim de Pesquisa e Desenvolvimento, 8).

BLETSOS, F. A Use of grafting and calcium cyanamide as alternatives to methyl bromide soil fumigation and their effects on growth, yield, quality and fusarium wilt control in melon. Journal of Phytopathology, Berlin, v.153, n.1, p. 155-161, 2005

CARDOSO, J. E. SANTOS, A. A.; ROSSETTI, A.G.; VIDAL, J.C. Relationship between incidence and severity of cashew gummosis in semiarid notheastern Brazil. Plant Pathology, London,v. 53, n.1, p. 363-367, 2004.

CARDOSO, J.E., FREIRE, F.C.O.; SÁ, F.T. Disseminação e controle da resinose em troncos de cajueiro decepados para substituição de copa. Fitopatologia Brasileira, Brasília, v.23, n.1, p. 48 - 50, 1998.

CARDOSO, J.E.; VIDAL, J.C.; SANTOS, A.A.; FREIRE, F.C.O.; VIANA, F.M.P. First report of black branch dieback of cashew by Lasiodiplodia theobromae in Brazil. Plant Disease, St. Paul, v. 86, n. 5 , p. $558,2002$.

CARDOSO, J.E.; PAIVA, J.R.; CAVALCANTI, J.J.V.; SANTOS, A.A.; VIDAL, J.C. Evaluation of resistance in dwarf cashew to gummosis in northeastern Brazil. Crop Protection, Oxford, v. 25, p. 855-859, 2006.
CARDOSO, J. E.; VIANA, F. M. P.; CYSNE, A.; FARIAS, F. C.; SOUSA, R. N. M. Clone Embrapa 51: uma alternativa para resistência à resinose-docajueiro. Fortaleza: Embrapa Agroindústria Tropical, 2007. (Comunicado Técnico, 130).

CARDOSO, J. E.; WILKINSON, M. J. Desenvolvimento e caracterização de marcadores microsatélites para o fungo Lasiodiplodia theobromae. Summa Phytopathologica, Botucatu, v.34, n.1, p.55-57, 2008.

CAVALCANTI JUNIOR, A. T.; CORREA, D.; BUENO, D. Propagação. In: Barros, L. M. (Ed.). Caju produção: aspectos técnicos. Fortaleza: Embrapa Agroindústria Tropical, 2002. p. 43-48. (Frutas do Brasil, 30).

CHAVES, R. C.; JUNQUEIRA, N. T. V.; MANICA, I. Enxertia de maracujazeiro-azedo em estacas herbáceas enraizadas de espécies de passifloras nativas. Revista Brasileira de Fruticultura, Jaboticabal, v. 26, n.1, p.120-123. 2004.

CICHY, K. A.; SNAPP, S. S.; KIRK, W. W. Fusarium root rot incidence and root system architecture in grafted common bean lines. Plant Soil, Dordrecht, v. 300, p. 233-244, 2007.

CILLIERS, A. A review of Lasiodiplodia theobromae with particular reference to its occurrence on coniferous seeds. South African Forest Journal, Pretoria, v. 166, p. 47-52, 1993.

COHEN R Horticultural and pathological aspects of Fusarium wilt management using grafted melons. HortScience, Alexandria, v. 37, p. $1069-1073$, 2002.

FAETH, S. H. Are endophytic fungi defensive plant mutualists? OIKOS, Lund, v. 98, p. 25-36, 2002.

FREIRE, F.C.O.; CARDOSO, J.E. Diseases of cashew nut plants (Anacardium occidentale L.) in Brazil. Crop Protection, Oxford, v. 21, p. $489-$ 494, 2002.

GIRARDI, E. A.; MOURÃO FILHO, F. A. A.; PIEDADE, S. M. S. Desenvolvimento vegetativo e custo de produção de porta-enxertos de citros em recipientes para fins de subenxertia. Pesquisa Agropecuária Brasileira, Brasília, v.42, n.5, p.679-687, 2007. 
LEE, J.M. Cultivation of grafted vegetables 1 . Current status, grafting methods, and benefits. HortScience, Alexandria, v. 29, p. 235-239, 1994.

MELETTI, L. M. M.; BRUCKNER, C. H. Melhoramento genético. In: BRUCKNER, C. H.; PICANÇO, M. C. (Ed.). Maracujá: tecnologia de produção, pós-colheita, agroindústria, mercado. Porto Alegre: Cinco Continentes, 2001. p. 345-385.

MENEZES, J. M. T.; OLIVEIRA, J. C.; RUGGIERO, C.; BANZATO, D. A. Avaliação da taxa de pegamento de enxertos de maracujá-amarelo sobre espécies tolerantes à "morte prematura de plantas". Científica, São Paulo, v.22, n.1, p.95-104, 1994.

MOHALI, S., BURGESS, T. I.; WINGFIELD, M. J. Diversity and host association of the tropical tree endophyte Lasiodiplodia theobromae revealed using simple sequence repeat markers. Forest Pathology, Berlin, v. 35, p. 385-396, 2005.

MORENO, P.; PIQUER, J.; NAVARRO, L.; CARBONELL, E. A.; PINA, J.A. Ensayos para evitar el decaimento causado por el vírus de la tristeza de los cítricos (CTV) en arboles injertados sobre patron 'Naranjo Amargo'. Investigaciones Agrarias y Producción Vegetal, Santiago, v.9, p.465-476, 1994.

MULLEN, J. M.; GILLIAM, C. H.; HAGAN, A. K.; MORGAN-JONES, G. Canker of dogwood caused by Lasiodiplodia theobromae, a disease influenced by droight stress or cultivar selection. Plant Disease, St. Paul, v. 75, p. 86-889. 1991.

PACE, C. A. M. Comparação de quatro métodos de enxertia para o maracujazeiro-amarelo Passiflora edulis f. flavicarpa Deg. In: CONGRESSO BRASILEIRO DE FRUTICULTURA, 7., 1983, Florianópolis. Anais... Florianópolis: EMPASC/Sociedade Brasileira de Fruticultura, 1984. p.983-988.
PAIVA, J. R.; CARDOSO, J. E.; CRISÓSTOMO, J. R.; CAVALCANTI, J. J .V.; ALENCAR, E. S. Clone de Cajueiro-Anão Precoce BRS 226 ou Planalto: Nova Alternativa para o Plantio na Região Semi-árida do Nordeste. Fortaleza: Embrapa Agroindústria Tropical, 2002. 4p. (Comunicado Técnico, 78).

PAIVA, J. R.; CARDOSO, J. E.; MESQUITA, A. L. M.; CAVALCANTI, J. J. V.; SANTOS, A. A. Desempenho de clones de cajueiro-anão precoce no semi-árido do Estado do Piauí. Ciência Agronômica, Fortaleza, v. 39, p. 295-300, 2008.

POMPEU JUNIOR, J. Porta-enxertos. In: MATTOS JUNIOR, D.; NEGRI, J. D.; PIO, R. M.; POMPEU JUNIOR, P. (Ed.). Citros. Campinas: Instituto Agronômico de Campinas, Fundag, 2005. p.63-94.

RUBINI, M. R.; SILVA-RIBEIRO, R. T.; POMELLA, A. W. V.; MAKI, C. S.; ARAÚJO, W. L.; SANTOS, D. R. D.; AZEVEDO, J. L. Diversity of endophytic fungal community of cacao (Theobroma cacao L.) and biological control of Crinipellis perniciosa, causal agent of Witches' Broom Disease. International Journal of Biological Sciences, New South Wales, v. 1, p. 24-33, 2005.

RUGGIERO, C. Situação da Cultura do Maracujazeiro no Brasil. Informe Agropecuário, Belo Horizonte, v.21, n.206, p.5-9, 2000.

SÃO JOSÉ, A. R. Propagação do Maracujazeiro. In: SÃO JOSÉ, A.R. (Ed.). A cultura do maracujá no Brasil. Jaboticabal: FUNEP, 1991. p.25-43.

SHAKED, A.; COHEN, A.; HAMOU, M.; HASDAI, D. Inarching of 'Swingle' citrumelo in Israel. HortScience, Alexandria, v.22, p.1258-1260, 1987. 